\title{
Functional, cognitive, and nutritional decline in 435 elderly nursing home residents after the first wave of the COVID-19 Pandemic
}

\author{
Patricia Pérez-Rodríguez ${ }^{1}$ Macarena Díaz de Bustamante ${ }^{1}$. Salvador Aparicio Mollá ${ }^{1}$ María Caridad Arenas ${ }^{1}$. \\ Susana Jiménez-Armero ${ }^{1}$ - Pilar Lacosta Esclapez ${ }^{2} \cdot$ Liliana González-Espinoza $^{3}$. Cristina Bermejo Boixareu ${ }^{1}$
}

Received: 28 December 2020 / Accepted: 31 May 2021 / Published online: 24 June 2021

(c) European Geriatric Medicine Society 2021

\section{Key summary points}

Aim To compare functional, cognitive, and nutritional status before and after COVID-19 pandemic among institutionalized older adults.

Findings High frequencies of functional, cognitive, emotional, and nutritional decline were found after the first wave of COVID19 pandemic independently of the infection.

Message Health workers and managers should take measures to prevent this decline in institutionalized elderly and its potential adverse effects.

\begin{abstract}
Purpose Many institutionalized older people have died during the first wave of COVID-19. Other related consequences have not yet been described objectively. The aim of this study was to compare functional, cognitive, and nutritional status before and after the first wave among nursing home residents, in both COVID-19 and non-COVID-19 patients.

Methods Older adults institutionalized in four nursing homes were assessed from May to June 2020, by a geriatric multidisciplinary team in collaboration with the nursing homes staff. Comprehensive geriatric assessment was performed including functional, cognitive, and nutritional variables before and after the first wave of the pandemic. Data from residents with positive results for microbiological testing for SARS-CoV-2 were compared with those who did not.

Results 435 nursing home residents were included. The median age was $86.77 \pm 8.5$ years, $78.4 \%$ were women. 190 (43.9\%) tested positive for coronavirus. Functional decline after the first wave was detected in $20.2 \%$ according to the Barthel Index and in $18.5 \%$ according to functional ambulation categories, $p<0.001$. Cognitive status worsened by 22 and $25.9 \%$ according to the global deterioration scale $(p<0.001)$ and Lobo's Mini-Examen Cognoscitivo $(p 0.01)$, respectively. Onset of depressive symptoms was found in $48 \%$ ( $p<0.001)$. The prevalence of malnutrition increased by 36.8 and $38.4 \%$ lost weight. When comparing the functional, cognitive, and nutritional decline between COVID-19 and non-COVID-19 patients no clinical or statistically significant differences were found except for the presence of prior malnutrition, higher in the COVID-19 group. Conclusion We observed a significative functional, cognitive, and nutritional decline in institutionalized elderly after the first wave of COVID-19. These results may be caused by the lockdown itself, since no differences have been found between COVID-19 and non-COVID-19 patients. According to these results, interventions are necessary during social isolation or confinement to prevent systemic decline in the elderly.
\end{abstract}

Keywords COVID $\cdot$ Elderly $\cdot$ Malnutrition $\cdot$ Nursing homes $\cdot$ Decline

Patricia Pérez-Rodríguez

ppr.patriciaperez@gmail.com

1 Geriatrics Department, Hospital Universitario Puerta de Hierro Majadahonda, C/ Joaquín Rodrigo, 2, Majadahonda, 28220 Madrid, Spain

2 Grupo Sergesa, Madrid, Spain

3 Grupo Albertia, Madrid, Spain

\section{Introduction}

The first wave of the COVID-19 pandemic presented an unprecedented public health problem, one that was especially devastating in older adults. This is demonstrated by the mortality figures: over $95 \%$ of deaths from COVID-19 
in Europe and over $80 \%$ of deaths in the United States and China have occurred in people aged 60 years or older [1].

COVID-19 shows multi-systemic involvement and, in the elderly, it presents a great complexity that requires a comprehensive and multidisciplinary approach adapted to the multiple pathologies of these patients [2]. Among the elderly, it is institutionalized older adults who present the highest rates of comorbidity, frailty, and disability [3]. Comorbidity and frailty have been shown to worsen the prognosis of SARSCoV-2 involvement [2, 4, 5].

It should be added that infection is easily transmitted in the nursing home environment [6], since they are built for communal living and staffed accordingly, making social distancing or isolation sometimes difficult or even impossible, thus favouring contagion [7]. In addition, clinical symptoms in the elderly are atypical, so detection is challenging [8]. Other factors that explain the enormous impact of the first wave of COVID-19 in nursing homes are the fact that not enough tests were done [7,9]; health professionals, their training, and protection measures were scarce [7].

We also know that the social isolation measures (fewer social interactions, restrictions in family visits, less physical activity), necessary to prevent the spread of the disease can have very serious consequences in older patients [7], especially if they are frail, such as functional and cognitive decline, depression, and increased mortality [2, 10], although collateral damage from isolation remains to be determined and has yet to be objectively described.

The pre-pandemic prevalence of functional dependence, cognitive decline, and malnutrition is much higher in institutionalized older adults compared to older adults in the community [3]. Despite the variability between territories, according to Spanish and European research, around $81 \%$ of the elderly who live in nursing homes are dependent [11, 12]. Functional impairment has been shown to be a predictor of mortality, even greater than comorbidity [13]. It is also known that in these people, confinement to a bed or immobilization facilitates an accelerated reduction in muscle mass, strength, and functional capacity.

The prevalence of dementia in nursing homes is around $68 \%$, of which $30 \%$ is severe [11]. Dementia in patients with COVID-19 represents a significant risk of mortality, falls, aspiration pneumonia, and risk of delirium [14]. The prevalence of depression in nursing homes nationwide is around $30 \%$ [11].

There is a high prevalence of malnutrition (30\%) and risk of malnutrition (50\%) in nursing homes [15]. Malnutrition is associated with multiple adverse events such as functional impairment, falls, fractures, greater severity of acute and chronic diseases, higher mortality [15], and immune dysfunction [16]. Malnutrition in patients with COVID-19 is high [17], and can be related with a longer and unfavourable recover $[18,19]$. The prevention, diagnosis and treatment of malnutrition must be included in the management of COVID-19 patients [18].

The main aim of this study is to compare the functional, cognitive, and nutritional status of institutionalized older adults before and after the first wave of the COVID-19 pandemic and also to assess whether there are differences between those who contracted COVID-19 and those who didn't.

\section{Methods}

The study was carried out by a multidisciplinary geriatric team at a tertiary hospital that has 613 hospital beds and 62 nursing homes in its area of influence, serving a total of 6200 residents.

A multicentre observational study was conducted in four of the nursing homes in this area between May 21 and June 23,2020 . All residents of these centres were included except those who were hospitalized or at home at the time of the study. A comprehensive geriatric assessment of each resident was performed in person by the hospital geriatric multidisciplinary team, which consisted of geriatricians, clinical nutritionists and nurses, with the collaboration of the nursing home health professionals (doctors, nursing staff and occupational therapists).

Demographic data were collected such as age and sex, and clinical data such as the presence or not of SARS-CoV-2 infection diagnosed by microbiological tests (PCR-Polymerase Chain Reaction and/or IgG/IgM rapid test). Functional, cognitive, and nutritional scale data were collected from the nursing home records prior to the start of the COVID-19 pandemic (between January and March 2020), and these same scales were used after the first wave of the pandemic, which occurred in Spain between the months of March and May 2020.

Functional assessment was carried out using the Barthel Index (BI) and the Functional Ambulation Categories (FAC) scale. BI scores were grouped as: independence for basic activities of daily living (BI 90-100), mild dependence (BI 60-89), moderate dependence (BI 40-59), severe dependence (BI 21-39) and total dependence (BI $\leq 20)$. A FAC score of 0 indicates the patient cannot walk or requires assistance from more than one person. FAC values of 1 , 2 , and 3, indicate that the patient can walk with great help from one-person, light help or supervision, respectively. A FAC value of 4 indicates independence for ambulation on level surfaces and a FAC value of 5 indicates independence on all surfaces.

Regarding cognitive variables, we used the global deterioration scale (GDS), a cognitive assessment scale that includes values from 1 (no cognitive decline) to 7 (severe cognitive decline); Lobo's Mini-Examen Cognoscitivo 
(MEC), which distinguishes the following categories: 35-25: no cognitive impairment, 24-20: mild cognitive impairment, 19-14: moderate cognitive impairment and $<14$ : severe cognitive impairment; and the 10-item short portable mental status questionnaire (Pfeiffer's SPMSQ), in which values equal to or greater than 3 errors are suggestive of cognitive impairment. To assess mood, the residents were asked two questions in relation to the last month: "¿Have you often felt sad, discouraged or hopeless?" And "Have you felt less interest or pleasure in doing things?" [20]. A positive response was indicative of depressive syndrome. In patients whose functional situation prevented them from responding adequately to this question, the nursing home staff were consulted about depressive symptoms.

Nutritional assessment was carried out using the Mini Nutritional Assessment-Short Form (MNA-SF) according to which three categories are established: malnutrition ( $0-7$ points), risk of malnutrition ( $8-11$ points) or no risk (12-14 points). The nursing home weight and height records were used to calculate body mass index (BMI), with postCOVID-19 weight measurements taken in patients for whom the records were not available. It was quantified by percentage how was each person's food intake before and after, according to the nursing home medical records.

Functional, cognitive, and nutritional decline were defined as a decline of at least one category of each variable during the first wave of the COVID-19 pandemic.

The results for the different variables before and after the pandemic were subsequently analysed by comparing those of the "COVID-19" cohort of residents who tested positive for SARS-CoV-2 from microbiological tests with those of the "non-COVID-19" cohort who were not infected.

After obtaining signed informed consent from each resident or a family member, the data were collected on anonymous data collection sheets, after which they were entered into the database. The study was approved by the Hospital's Ethics Committee.

\section{Statistical analysis}

A descriptive analysis of the categorical variables was performed using absolute and relative frequencies. For numerical variables, the mean and standard deviation or the median and the IQR were used according to normality of distribution.

Changes between the scores of the categorical variables were compared using McNemar's test; for numerical variables, the Wilcoxon signed-rank test was used.

We looked for differences in certain variables between COVID-19 and non-COVID-19 patients using the Chisquare test to compare categorical variables and the Mann-Whitney $U$ test for numerical variables. Subsequently, an ANCOVA analysis was performed to evaluate whether infection with COVID-19 was associated with worse results, adjusting for the previous score of each resident.

Linear or logistic regressions were performed depending on the variable evaluated. The level of statistical significance was set at 0.05 .

The statistical software used was Stata/IC v.16 (StataCorp. 2019. Stata Statistical Software: Release 16. College Station, TX: StataCorp LLC.)

\section{Results}

The study included 435 residents from 4 different nursing homes $(69,75,118$, and 173 , respectively). The mean age of people included in this study was $86.77 \pm 8.5$ years. Of these patients, 341 (78.4\%) were women. There were 191 (43.9\%) who had presented positive in microbiological tests for COVID-19. The median of the Charlson Index was 3 points (IQR 2-4). In March, 646 residents were in these nursing homes, 175 (27\%) died during the first wave and 36 were not present in the moment of the study.

The functional, cognitive, and nutritional variables before and after the first wave are listed in Table 1. The largest decline noted was in the group of higher degree of severity in the activities of daily living $(\mathrm{BI}<20 / 100)$ (from 29.2 to $39.3 \%$ ). In the FAC test was found the highest increase also in the non-functional ambulation group (from 36.6 to $40 \%$ ). In the cognitive assessment of nursing home residents advanced dementia was the most prevalent group (42.5, 37.3 and $54.2 \%$, respectively, according to GDS, MEC and Pfeiffer's SPMSQ) after the first wave, but only in the MEC and the Pfeiffer's SPMSQ tests were detected changes. Most striking increase was found in the prevalence of depression (from 11.1 to $54.5 \%$ ) and malnutrition according to the MNA-SF (from 20 to 56.8\%) (Fig. 1).

The analysis included the number and percentage of subjects who presented a decline of at least one category for each variable during the first wave of the COVID-19 pandemic, adjusted for the previous status (Table 2). The decline was statistically significant for all variables analysed except for Pfeiffer's SPMSQ.

In the functional analysis $20.2 \%$ of residents presented a decline of at least one category in the Barthel Index and $18,5 \%$ in the FAC test. There is a greater functional impairment in the group of older adults with moderate (BI 40-59: $32.4 \%$ ) and severe dependence (BI 21-39: 44.1\%). Fit patients (Barthel 90-100 and FAC 5) were the groups with less functional impairment in both functional scales.

Cognitive impairment was observed in $22,5 \%$ of subjects according to GDS, $25,9 \%$ to MEC and $14,1 \%$ in Pfeiffer's SPMSQ, predominantly happened in those with mild (GDS 3) and moderate cognitive impairment (GDS 4, MEC 20-23) 
Table 1 Functional, cognitive, and nutritional variables of residents before and after the first wave of the COVID-19 pandemic $(n=435)$

\begin{tabular}{|c|c|c|c|c|}
\hline \multirow[b]{3}{*}{ Barthel index } & \multicolumn{2}{|c|}{ Prior to March 2020} & June 2020 & \multirow[t]{2}{*}{$p$} \\
\hline & $n$ & & & \\
\hline & 435 & $45(20 ; 75)$ & $35(10 ; 70)$ & $<0.001$ \\
\hline $0-20$ & & $127(29.2 \%)$ & $171(39.3 \%)$ & \\
\hline $21-39$ & & $68(15.6 \%)$ & $54(12.4 \%)$ & \\
\hline $40-59$ & & $68(15.6 \%)$ & $68(15.6 \%)$ & \\
\hline $60-89$ & & $131(30.1 \%)$ & $102(23.5 \%)$ & \\
\hline $90-100$ & & $41(9.4 \%)$ & $40(9.2 \%)$ & \\
\hline FAC & 426 & $2(0 ; 4)$ & $1(0 ; 4)$ & $<0.001$ \\
\hline 0 & & $143(33.6 \%)$ & $172(40.0 \%)$ & \\
\hline $1-2$ & & $72(16.9 \%)$ & $84(19.5 \%)$ & \\
\hline 3 & & $63(14.8 \%)$ & $52(12.1 \%)$ & \\
\hline $4-5$ & & $148(34.7 \%)$ & $122(28.4 \%)$ & \\
\hline GDS & 134 & $6(3 ; 7)$ & $6(3 ; 7)$ & $<0.001$ \\
\hline 1 & & $22(16.4 \%)$ & $16(12.2 \%)$ & \\
\hline $2-3$ & & $20(14.9 \%)$ & $22(16.8 \%)$ & \\
\hline $4-5$ & & $19(14.2 \%)$ & $22(16.8 \%)$ & \\
\hline $6-7$ & & $73(54.5 \%)$ & $71(54.2 \%)$ & \\
\hline MEC & 109 & $24.5(16 ; 31)$ & $22(13 ; 29)$ & $<0.001$ \\
\hline $0-14$ & & $23(21.1 \%)$ & $33(30 \%)$ & \\
\hline $15-19$ & & $15(13.8 \%)$ & $16(14.55 \%)$ & \\
\hline $20-23$ & & $17(15.6 \%)$ & $20(18.2 \%)$ & \\
\hline $24-35$ & & $54(50 \%)$ & $41(37.3 \%)$ & \\
\hline Pfeiffer's SPMSQ & 251 & $6(3 ; 9)$ & $7(3 ; 10)$ & $<0.001$ \\
\hline $0-2$ & & $57(22.7 \%)$ & $58(22.4 \%)$ & \\
\hline $3-4$ & & $30(12 \%)$ & $31(12 \%)$ & \\
\hline $5-7$ & & $72(28.7 \%)$ & $60(23.2 \%)$ & \\
\hline $8-10$ & & $92(36.7 \%)$ & $110(42.5 \%)$ & \\
\hline Depression & 414 & $46(11.1 \%)$ & $214(53.5 \%)$ & \\
\hline MNA-SF & 435 & $10(8 ; 12)$ & $7(5 ; 9)$ & $<0.001$ \\
\hline Normal & & $127(29.2 \%)$ & $11(2.5 \%)$ & \\
\hline Risk of malnutrition & & $221(50.8 \%)$ & $177(40.7 \%)$ & \\
\hline Malnutrition & & $87(20 \%)$ & $247(56.8 \%)$ & \\
\hline Body mass index & 435 & $24.8(21.6 ; 28.4)$ & $23.6(20.7 ; 27.4)$ & $<0.001$ \\
\hline$<18.5 \mathrm{~kg} / \mathrm{m}^{2}$ & & $40(9.2 \%)$ & $57(13.1 \%)$ & \\
\hline $18.5-24.9 \mathrm{~kg} / \mathrm{m}^{2}$ & & $192(44.1 \%)$ & $212(48.7 \%)$ & \\
\hline $25-29.9 \mathrm{~kg} / \mathrm{m}^{2}$ & & $131(30.1 \%)$ & $109(25.1 \%)$ & \\
\hline$>30 \mathrm{~kg} / \mathrm{m}^{2}$ & & $72(16.6 \%)$ & $57(13.1 \%)$ & \\
\hline$\%$ of food intake & 435 & & & $<0.001$ \\
\hline $25 \%$ & & $2(0.5 \%)$ & $8(1.8 \%)$ & \\
\hline $50 \%$ & & $5(1.2 \%)$ & $55(12.6 \%)$ & \\
\hline $75 \%$ & & $61(14 \%)$ & $147(33.8 \%)$ & \\
\hline $100 \%$ & & $367(84.4 \%)$ & $225(51.7 \%)$ & \\
\hline
\end{tabular}

Valid cases: number of cases with valid data for each variable

$F A C$ functional ambulation category scale, GDS global deterioration scale, MEC Lobo's Mini-Examen Cognoscitivo, Pfeiffer's SPMSQ short portable mental status questionnaire, MNA-SF mini nutritional assessment short-form, $B M I$ body mass index prior to the pandemic. Onset of depression after the first wave of the pandemic appeared in 164 of $344(47.97 \%)$ residents who did not have prior depression $(p<0.001)$.
In the nutritional assessment there was a worsening in $92.9 \%$ of patients whose previous nutritional situation was considered normal according to the MNA-SF and a 
FUNCTIONAL DECLINE

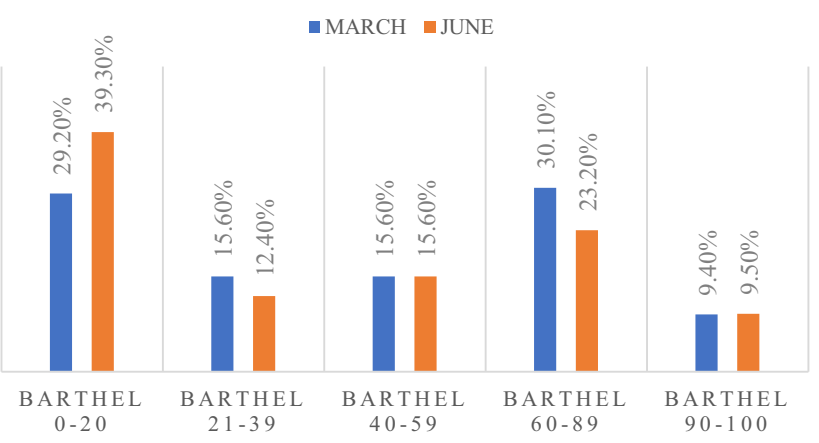

DEPRESSION

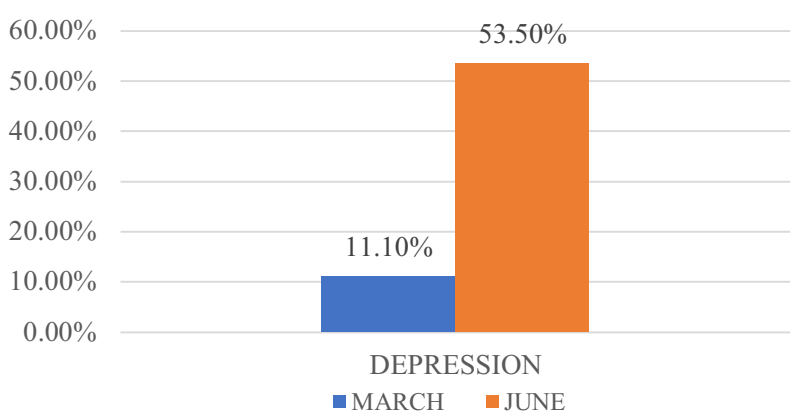

COGNITIVE STATUS (MEC)
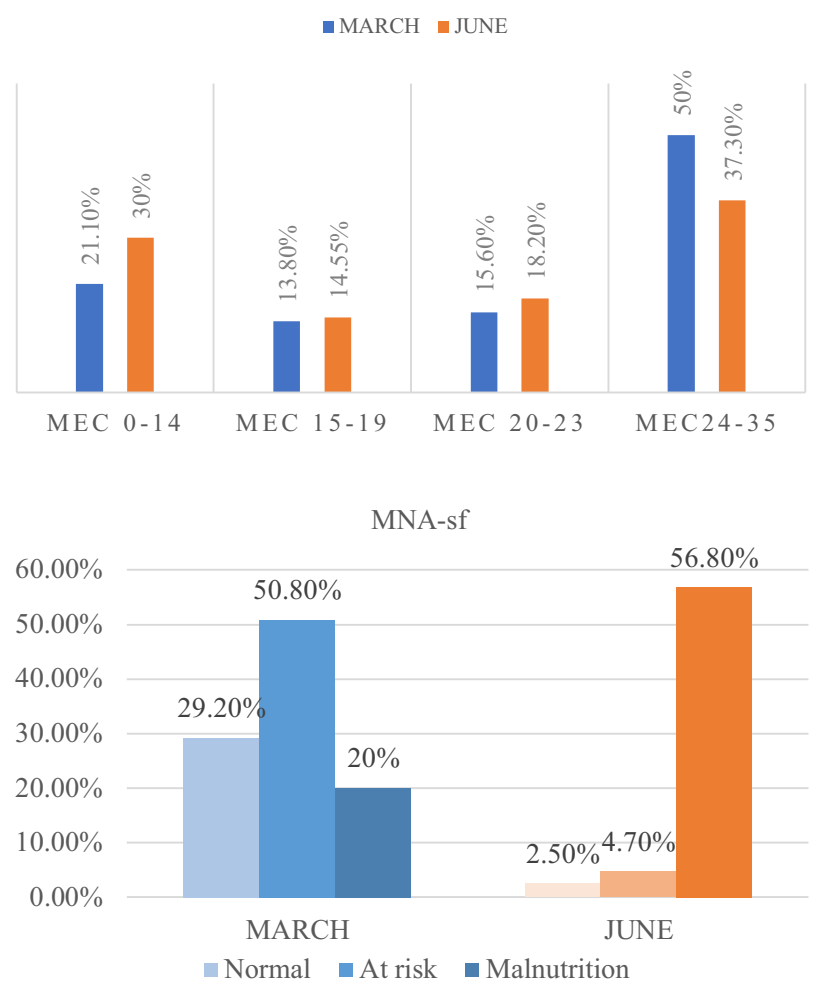

Fig. 1 Functional, cognitive and nutritional variables of residents before and after the first wave of the COVID-19 pandemic ( $n=435)$

worsening of $63.8 \%$ of those at risk of malnutrition after the first wave of the COVID-19 pandemic. Likewise, the greatest decrease in the percentage of food intake occurred in patients with food intakes greater than $75 \%$ prior to the first wave. Weight loss was observed in 167 residents (38.4\%), the median weight loss was $2.1 \mathrm{~kg}$ (IQR 0.4-4.6).

The second aim of the study was to compare functional, cognitive, and nutritional decline between residents that had suffered COVID-19 during the first wave and residents free from SARS-CoV infection.

We compared baseline characteristics of the COVID19 cohort versus the non-COVID-19 cohort (Table 3), and there were no statistically significant differences analysing demographic variables (age, sex), neither Charlson index, functional and cognitive variables, nor depressive symptoms. The only difference detected was in the nutritional assessment according to the MNA-SF before March, 80.1\% of the COVID-19 patients identified malnutrition or risk of malnutrition compared to $63.5 \%$ of the non-COVID-19 residents ( $p$ 0.001).

No statistically significant differences were detected when studying functional impairment in both groups (Barthel Index or FAC). There were also no differences in terms of cognitive decline according to MEC and Pfeiffer. In respect to GDS, COVID-19 patients had, on average, a GDS score after the first wave of the pandemic 0.27 points higher than that of non-COVID-19 residents, adjusting for the previous GDS score of each resident (95\% CI 0.07; $0.46, p$ value 0.007).

In relation to depressive syndrome, subjects with COVID19 had a $43 \%$ lower risk of developing depression after the pandemic (OR 0.57, 95\% CI 0.37; 0.88, $p$ value 0.001 ).

Assessing nutritional status, no significant differences were found in terms of weight loss or BMI. However, COVID-19 patients presented scores in the MNA-SF that were 0.44 points lower, adjusting for previous scores, than those of non-COVID-19 patients (95\% CI $-0.8 ;-0.04, p$ $0.03)$.

\section{Discussion}

The findings of this study show that institutionalized older adults presented significant worsening in their functional, cognitive, emotional, and nutritional status after the first wave of the pandemic, regardless of whether they were infected by SARS-CoV-2 or not, since no clinically or statistically significant differences were found when comparing the decline data for both groups. This shows that the observed worsening is not only associated to infection but 
Table 2 Residents who presented a decline of at least one category for each variable during the first wave of the COVID-19 pandemic, adjusted for the previous status $(n=435)$

\begin{tabular}{|c|c|c|c|}
\hline & $N$ & $n(\%)$ & $p$ \\
\hline \multicolumn{4}{|l|}{ Previous Barthel index } \\
\hline $90-100$ & 41 & $4(9.8 \%)$ & \\
\hline $60-89$ & 131 & $32(24.4 \%)$ & \\
\hline $40-59$ & 68 & $22(32.4 \%)$ & \\
\hline $21-39$ & 68 & $30(44.1 \%)$ & \\
\hline Total & 435 & $88(20.2 \%)$ & $<0.001$ \\
\hline \multicolumn{4}{|l|}{ Previous FAC } \\
\hline 1 & 29 & $8(27.6 \%)$ & \\
\hline 2 & 43 & $13(30.2 \%)$ & \\
\hline 3 & 63 & $23(36.5 \%)$ & \\
\hline 4 & 90 & $27(30 \%)$ & \\
\hline 5 & 58 & $8(13.8 \%)$ & \\
\hline Total & 426 & $79(18.5 \%)$ & $<0.001$ \\
\hline \multicolumn{4}{|l|}{ Previous GDS } \\
\hline 1 & 22 & $7(31.8 \%)$ & \\
\hline 2 & 11 & $2(18.2 \%)$ & \\
\hline 3 & 9 & $4(44.4 \%)$ & \\
\hline 4 & 8 & $6(75 \%)$ & \\
\hline 5 & 10 & $4(40 \%)$ & \\
\hline 6 & 28 & $5(17.9 \%)$ & \\
\hline Total & 127 & $28(22 \%)$ & $<0.001$ \\
\hline \multicolumn{4}{|l|}{ Previous MEC } \\
\hline $15-19$ & 15 & $6(40 \%)$ & \\
\hline $20-23$ & 17 & $7(41.2 \%)$ & \\
\hline $24-35$ & 54 & $15(27.8 \%)$ & \\
\hline Total & 108 & $28(25.9 \%)$ & 0.01 \\
\hline \multicolumn{4}{|c|}{ Previous Pfeiffer's SPMSQ } \\
\hline $0-2$ & 57 & $11(19.3 \%)$ & \\
\hline $3-4$ & 29 & $8(27.6 \%)$ & \\
\hline $5-7$ & 70 & $16(22.9 \%)$ & \\
\hline Total & 248 & $35(14.1 \%)$ & 0.83 \\
\hline \multicolumn{4}{|l|}{ Previous MNA-SF } \\
\hline Normal & 126 & $117(92.9 \%)$ & \\
\hline Risk of malnutrition & 221 & $141(63.8 \%)$ & \\
\hline Total & 435 & $258(59.3 \%)$ & $<0.001$ \\
\hline \multicolumn{4}{|l|}{ Previous BMI } \\
\hline $18.5-24.9 \mathrm{~kg} / \mathrm{m}^{2}$ & 192 & $20(10.4 \%)$ & \\
\hline $25-29.9 \mathrm{~kg} / \mathrm{m}^{2}$ & 131 & $44(33.6 \%)$ & \\
\hline$>30 \mathrm{~kg} / \mathrm{m}^{2}$ & 72 & $19(26.4 \%)$ & \\
\hline Total & 435 & $83(19.1 \%)$ & $<0.001$ \\
\hline \multicolumn{4}{|l|}{$\%$ Previous intake } \\
\hline $75 \%$ & 61 & $28(45.9 \%)$ & \\
\hline $100 \%$ & 367 & $142(38.8 \%)$ & \\
\hline Total & 435 & $170(39.1 \%)$ & $<0.001$ \\
\hline
\end{tabular}

$B I$ Barthel index, FAC functional ambulation category scale, GDS global deterioration Scale, MEC Lobo's Mini-Examen Cognoscitivo, Pfeiffer's SPMSQ short portable mental status questionnaire, $M N A$ $S F$ mini nutritional assessment short-form, BMI body mass index, Total total of valid cases for each variable is probably related to isolation during the first wave of the pandemic.

It is known that functional [21, 22] and cognitive [23] decline happen in elderly especially in those institutionalized compared with noninstitutionalized and there is a huge interpersonal variability depending on the characteristics and comorbidities of individuals. A drop on MMSE score of 2.8 points after placement in a NH is described [23]. Regarding functional decline, it is observed in $34.95 \%$ of institutionalized after 10 months [21] and in $5.8 \%$ of institutionalized during 6 months [22].

After the pandemic, worryingly high frequencies of severe or total dependence, severe dementia, depression, and malnutrition were detected in this population group, which has important implications for the Health Care System. Isolation has led to a worsening global condition in the elderly that is avoidable and could be considered iatrogenic.

It is striking that functional, cognitive, and nutritional loss is greater in patients with mild-moderate impairments or those at risk, which indicates that this is the group that we must focus on the most to prevent potential complications during the second wave of COVID- 19.

Physical exercise, cognitive stimulation, and adequate nutritional management have disappeared in recent months due to the breakout of COVID-19 in nursing homes, which has stood out for its high rates of mortality and contagion. Confinement has favoured situations of prolonged immobility, little physical exercise, lack of cognitive stimulation [24, 25], anxiety [26], and depression [27]. According to our study, those who had COVID-19 may have lower risk to present depressive symptoms after the pandemic. These findings may be in relation to a better understanding of the confinement, since they passed the disease. Social isolation determines secondary functional decline, cognitive decline [28], and mood deterioration [24, 26, 27], as well as a negative impact at the nutritional level [19, 29], favouring a reduction in food intake and triggering a loss of muscle mass that is hard to recover by dietary modifications alone.

We detected statistically significant differences in nutritional status according to MNA-SF, which was worse in patients who presented COVID-19. This is consistent with published studies [17, 18], and warrants further investigation.

According to the results obtained in this study in relation to the high prevalence and risk of malnutrition, it is necessary to optimize nutritional intake through stimulation techniques, oral supplementation, and dedicating more time and trained caregivers to this matter.

These results, as well as the well-known mortality, should promote the search for different interventions or strategies at all levels so that health workers, managers and politicians may prevent, as far as possible, these results from being repeated and progressing if this infection persists worldwide 
Table 3 Baseline characteristics (pre-pandemic) of "COVID-19" and "non-COVID-19" cohorts. Data shown as median (IQR) and $n(\%)$

\begin{tabular}{|c|c|c|c|}
\hline$n=435$ & COVID-19 $(n=191)$ & Non-COVID-19 $(n=244)$ & $p$ \\
\hline Age (years) & $88(83 ; 92)$ & $88(83.5 ; 92)$ & 0.334 \\
\hline Sex & & & 0.594 \\
\hline Female & $152(79.6 \%)$ & $189(77.5 \%)$ & \\
\hline Barthel index & $40(20 ; 70)$ & $47.5(20 ; 75)$ & 0.042 \\
\hline $90-100$ & $15(7.9 \%)$ & $26(10.7 \%)$ & \\
\hline $60-89$ & $50(26.2 \%)$ & $81(33.2 \%)$ & \\
\hline $40-59$ & $31(16.2 \%)$ & $37(15.2 \%)$ & \\
\hline $21-39$ & $31(16.2 \%)$ & $37(15.2 \%)$ & \\
\hline $0-20$ & $64(33.5 \%)$ & $63(25.8 \%)$ & \\
\hline FAC & $2(0 ; 4)$ & $3(0 ; 4)$ & 0.224 \\
\hline 0 & $74(39.4 \%)$ & $69(29 \%)$ & \\
\hline $1-2$ & $24(12.8 \%)$ & $48(20.2 \%)$ & \\
\hline 3 & $24(12.8 \%)$ & $39(16.4 \%)$ & \\
\hline $4-5$ & $66(35.1 \%)$ & $82(34.5 \%)$ & \\
\hline GDS & $5(2 ; 6)$ & $6(3.5 ; 7)$ & 0.004 \\
\hline 1 & $15(22.7 \%)$ & $7(10.3 \%)$ & \\
\hline $2-3$ & $10(15.2 \%)$ & $10(14.8 \%)$ & \\
\hline $4-5$ & $14(21.2 \%)$ & $5(7.4 \%)$ & \\
\hline $6-7$ & $27(40.9 \%)$ & $46(67.6 \%)$ & \\
\hline MEC & $25(15 ; 31)$ & $24(18 ; 31)$ & 0.803 \\
\hline $0-14$ & $15(22.4 \%)$ & $8(19.1 \%)$ & \\
\hline $15-19$ & $10(14.9 \%)$ & $5(11.9 \%)$ & \\
\hline $20-23$ & $8(11.9 \%)$ & $9(21.4 \%)$ & \\
\hline $24-35$ & $34(50.8 \%)$ & $20(47.6 \%)$ & \\
\hline Pfeiffer's SPMSQ & $6(2 ; 9)$ & $6(3 ; 9)$ & 0.341 \\
\hline $0-2$ & $23(27.7 \%)$ & $34(20.2 \%)$ & \\
\hline $3-4$ & $11(13.3 \%)$ & $19(11.3 \%)$ & \\
\hline $5-7$ & $20(24.1 \%)$ & $52(31 \%)$ & \\
\hline $8-10$ & $29(34.9 \%)$ & $63(37.5 \%)$ & \\
\hline Depression & & & 0.115 \\
\hline No & $155(86.1 \%)$ & $213(91 \%)$ & \\
\hline Yes & $25(13.9 \%)$ & $21(9 \%)$ & \\
\hline MNA-SF & $9(8 ; 11)$ & $11(9 ; 12)$ & $<0.001$ \\
\hline Normal & $38(19.9 \%)$ & $89(36.5 \%)$ & \\
\hline Risk of malnutrition & $108(56.5 \%)$ & $113(46.3 \%)$ & \\
\hline Malnutrition & $45(23.6 \%)$ & $42(17.2 \%)$ & \\
\hline BMI & $24.9(21.9 ; 28.9)$ & $24.4(21.6 ; 28.1)$ & 0.156 \\
\hline$<18.5 \mathrm{~kg} / \mathrm{m}^{2}$ & $16(8.4 \%)$ & $24(9.8 \%)$ & \\
\hline $18.5-24.9 \mathrm{~kg} / \mathrm{m}^{2}$ & $80(41.9 \%)$ & $112(45.9 \%)$ & \\
\hline $25-29.9 \mathrm{~kg} / \mathrm{m}^{2}$ & $60(31.4 \%)$ & $71(29.1 \%)$ & \\
\hline$>30 \mathrm{~kg} / \mathrm{m}^{2}$ & $35(18.3 \%)$ & $37(15.2 \%)$ & \\
\hline$\%$ Food intake & & & 0.090 \\
\hline $25 \%$ & $1(0.5 \%)$ & $1(0.4 \%)$ & \\
\hline $50 \%$ & $3(1.6 \%)$ & $2(0.8 \%)$ & \\
\hline $75 \%$ & $36(18.9 \%)$ & $25(10.3 \%)$ & \\
\hline $100 \%$ & $151(79.1 \%)$ & $216(88.5 \%)$ & \\
\hline
\end{tabular}

Valid cases: number of cases with valid data for each variable

$F A C$ functional ambulation category scale, GDS global deterioration scale, MEC Lobo's Mini-Examen Cognoscitivo, Pfeiffer's SPMSQ short portable mental status questionnaire, MNA-SF mini nutritional assessment short-form, $B M I$ body mass index 
$[9,10,30]$. Failure to perform an adequate intervention would have a great impact on the health of our institutionalized elderly with great associated costs down the road [10].

Among the strengths of this study we can highlight that it is a multicentre study with a large sample $(n=435)$, including all the older adults institutionalized in those centres at the time of the study, without exclusion criteria, comparing both COVID-19 and non-COVID-19 cohorts. The study was carried out in person by a hospital multidisciplinary team using an individualized comprehensive geriatric assessment. There are no studies published to date with objective data on the consequences of isolation in nursing home residents.

Among the limitations of the study, it should be noted that the baseline variables were collected retrospectively from the nursing home records, so that on some occasions the initial assessments were performed by different people than those who performed them at the time of the study. The study did not include hospitalized patients, probably in serious condition, who were not at the nursing homes at the time of the study or those who died during the first wave. Since this is an observational study, these results cannot be considered conclusive nor can they be extrapolated to the general population, and therefore, further studies are necessary. In addition, we must study how much of the lost function can be recovered and how long it takes to recover.

\section{Conclusion}

We observed a significant impact in functional, cognitive, and nutritional status in older adults living in nursing homes after the first wave of the COVID-19 pandemic. This was not only conditioned by the infection itself, but also by the imposed isolation ${ }^{29}$. Maintaining ambulation and functional and cognitive stimulation, family support, and social interaction with other residents is essential to avoid dependence and depression, and to guarantee an adequate state of health [31]. Likewise, guaranteeing adequate nutritional intake would be a priority in the face of this second wave of COVID-19. In this situation of new normality, it is essential to develop the implementation of new technologies and to promote the use of apps to stimulate the patient at all levels [32], and to use telemedicine to optimize care for the elderly and avoid the general decline derived from COVID-19, confinement and social isolation. Further research aimed at the prevention of functional, cognitive, and nutritional deterioration of the elderly in the face of the COVID-19 pandemic is urgent.

Acknowledgements We thank all the health workers (doctors, nurses and medical assistants) as well as the leaders of the nursing homes for allowing us to carry out this study and collaborate in it. We thank Ana Royuela for the collaboration in the data analysis.
Author contributions $\mathrm{PPR}, \mathrm{MDB}, \mathrm{SAM}$ and $\mathrm{CBB}$ conceived and designed the study. PPR, MDB, SAM, MCA, SJA, PLE and LGE collected the data. PPR, MDB and $\mathrm{CBB}$ contributed to the literature review. PPR, MDB, MCA and $\mathrm{CBB}$ interpreted data. PPR, MDB and $\mathrm{CBB}$ were involved in the manuscript preparation. PPR, MDB, SAM, MCA, SJA, PLE, LGE and CBB critically reviewed the report, and all read and approved the final version.

Funding No funding was reported. The authors have no relevant financial or non-financial interests to disclose.

Availability of data and materials Data are available from the corresponding author upon reasonable request.

\section{Declarations}

Conflict of interest The authors have declared no conflict of interest for this article and no financial conflicts.

Ethical approval The study was approved by the Hospital's Ethics Committee.

Informed consent The patients or their families, gave consent to participate in the study.

\section{References}

1. Pan American Health Organization (2020) The Impact of COVID19 on older persons. https://www.paho.org/en/documents/policybrief-impact-covid-19-older-persons. Accessed 20 Jun 2020

2. Morley JE, Vellas B (2020) Editorial: COVID-19 and Older Adults. J Nutr Health Aging 24:364-365

3. Amblàs-Novellas J, Santaeugènia SJ, Vela E et al (2020) What lies beneath: a retrospective, population-based cohort study investigating clinical and resource-use characteristics of institutionalized older people in Catalonia. BMC Geriatr 20:187

4. Hägg S, Jylhävä J, Wang Y et al (2020) Age, frailty and comorbidity as prognostic factors for short-term outcomes in patients with COVID-19 in geriatric care. J Am Med Dir Assoc. https://doi.org/ 10.1016/j.jamda.2020.08.014

5. Hewitt J, Carter B, Vilches-Moraga A et al (2020) The effect of frailty on survival in patients with COVID-19 (COPE): a multicentre, European, observational cohort study. Lancet Public Health 5:e444-e451

6. McMichael TM, Currie DW, Clark S et al (2020) Epidemiology of covid-19 in a long-term care facility in King County. Washington N Engl J Med 382:2005-2011

7. Burki T (2020) England and Wales see 20000 excess deaths in care homes. Lancet 395(10237):1602

8. Knopp P, Miles A, Webb TE, a, (2020) Presenting features of COVID-19 in older people: relationships with frailty, inflammation and mortality. Eur Geriatr Med. https://doi.org/10.1007/ s41999-020-00373-4

9. Martinez-Peromingo J, Serra-Rexach JA (2020) Long-term care facilities and the COVID-19 $\mathrm{s}$ in Madrid. J Am Geriatr Soc. https://doi.org/10.1111/jgs.16665

10. Flatharta T, Mulkerrin EC (2020) Back to basics: giant challenges to addressing Isaac's "Geriatric Giants" post COVID-19 crisis. J Nutr Health Aging 24:705-707

11. Onder G, Carpenter I, Finne-Soveri H et al (2012) Assessment of nursing home residents in Europe: the Services and Health for 
Elderly in Long TERm care (SHELTER) study. BMC Health Serv Res 12:5

12. Serrano-Urrea R, Gómez-Rubio V, Palacios-Ceña D et al (2017) Individual and institutional factors associated with functional disability in nursing home residents: An observational study with multilevel analysis. PLoS ONE 12:e183945

13. Landi F, Liperoti R, Russo A et al (2010) Disability, more than multimorbidity, was predictive of mortality among older persons aged 80 years and older. J Clin Epidemiol 63:752-759

14. Bianchetti A, Rozzini R, Guerini F et al (2020) Clinical presentation of COVID19 in dementia patients. J Nutr Health Aging 24:560-562

15. Vaca Bermejo R, Ancizu García I, Moya Galera D et al (2014) Prevalencia de desnutrición en personas mayores institucionalizadas en España: un análisis multicéntrico nacional [Prevalence of malnutrition in Spanish institutionalized older people: a multicentric nationwide analysis]. Nutr Hosp 31:1205-1216

16. Handu D, Moloney L, Rozga $M$ et al (2020) Malnutrition care during the COVID-19 pandemic: considerations for registered dietitian nutritionists. J Acad Nutr Diet. https://doi.org/10.1016/j. jand.2020.05.012

17. Li T, Zhang Y, Gong C et al (2020) Prevalence of malnutrition and analysis of related factors in elderly patients with COVID-19 in Wuhan. China Eur J Clin Nutr 74:871-875

18. Barazzoni R, Bischoff SC, Breda J et al (2020) ESPEN expert statements and practical guidance for nutritional management of individuals with SARS-CoV-2 infection. Clin Nutr 39:1631-1638

19. Azzolino D, Saporiti E, Proietti M et al (2020) Nutritional considerations in frail older patients with COVID-19. J Nutr Health Aging 24:696-698

20. Arroll B, Khin N, Kerse N (2003) Screening for depression in primary care with two verbally asked questions: cross sectional study. BMJ 327:1144

21. Ang YH, Au SY, Yap LK et al (2006) Functional decline of the elderly in a nursing home. Singapore Med J 47(3):219-224

22. Yeh KP, Lin MH, Liu LK et al (2014) Functional decline and mortality in long-term care settings: static and dynamic approach. J Clin Gerontol Geriatr 5(1):13-17

23. González-Colaço Harmand M, Meillon C, Rullier L et al (2014) Cognitive decline after entering a nursing home: a 22 Year followup study of institutionalized and noninstitutionalized elderly people. J Am Med Dir Assoc 15(7):504-508
24. Kimura M, Ojima T, Ide K et al (2020) Allaying post-COVID19 negative health impacts among older people: the "Need To Do Something With Others"-Lessons From the Japan Gerontological Evaluation Study. Asia Pac J Public Health 16:1010539520951396

25. Gutiérrez Rodríguez J, Montero Muñoz J, Jiménez Muela F et al (2020) Variables associated with mortality in a selected sample of patients older than 80 years and with some degree of functional dependence hospitalized for COVID-19 in a Geriatrics Service. Rev Esp Geriatr Gerontol 55:317-325

26. Pierce M, Hope H, Ford T et al (2020) Mental health before and during the COVID-19 pandemic: a longitudinal probability sample survey of the UK population. Lancet Psychiatry 7:883-892

27. El M, Altintas E, Chapelet G et al (2020) High depression and anxiety in people with Alzheimer's disease living in retirement homes during the covid-19 crisis. Psychiatry Res 291:113294

28. Blanco-Tarrio E, Blanco Sánchez G (2020) Primary care, residential homes for the elderly, and COVID-19. SEMERGEN 46(Suppl 1):26-34

29. Cocuzzo B, Wrench A, O'Malley C (2020) Balancing Protection from COVID-19 and the Need for Human Touch in Nursing Homes. J Am Geriatr Soc. https://doi.org/10.1111/jgs.16861

30. Gordon AL, Goodman C, Achterberg W et al (2020) Commentary: COVID in care homes-challenges and dilemmas in healthcare delivery. Age Ageing 24(5):701-705

31. Yang Y-C, Chou C-L, Kao C-L (2020) Exercise, nutrition, and medication considerations in the light of the COVID pandemic, with specific focus on geriatric population. J Chin Med Assoc. https://doi.org/10.1097/jcma.0000000000000393

32. Su Z, Meyer K, Li Y et al (2020) Technology-based interventions for nursing home residents: implications for nursing home practice amid and beyond the influence of COVID-19: a systematic review protocol. Res Sq 18:56102

Publisher's Note Springer Nature remains neutral with regard to jurisdictional claims in published maps and institutional affiliations. 